\title{
ON THE STOPAGE RULE IN SOLUTION FOR MONOTONE ILL-POSED PROBLEMS
}

\author{
NGUYEN BUONG
}

\begin{abstract}
The purpose of this note is to present an iterative method for solving a regularized equation for nonlinear monotone ill-posed problems in Banach space and to study its stopage rule so that iteration sequence converges to a solution of initial problem, as the noisy data of the right-hand side converges to its exact.
\end{abstract}

\section{INTRODUCTION}

Let $X$ be a real reflexive Banach space and $X^{*}$ be its dual space. For the sake of simplicity norms of $X$ and $X^{*}$ will be denoted by one symbol $\|\cdot\|$. Let $A$ be a continuous and monotone operator with domain of definition $D(A)=X$ and range $R(A) \subseteq X^{*}$. Let $f_{0}$ be an element of $R(A)$.

Consider the nonlinear ill-posed problem.

$$
A(x)=f_{0} .
$$

By ill-posedness we mean that solution of (1.1) do not depend continuously on the data $f_{0}$. To solve (1.1) we can use variational method of Tikhonov regularization that consists of minimizing the functional

$$
\left\|A(x)-f_{\delta}\right\|^{2}+\alpha \Omega(x) \text {, over } D(A),
$$

where $\Omega(x)$ is a some functional that plays the role of regularization, $\alpha>0$ is a small parameter and the noisy data $f_{\delta}$ satisfies the condition

$$
\left\|f_{0}-f_{\delta}\right\| \leq \delta \rightarrow 0 .
$$

In [1] and [2] they showed another version of Tikhonov regularization, that is the use of regularized equation

$$
A(x)+\alpha U(x)=f_{\delta}
$$

Supported by the National Program for Fundamental Researches in Natural Sciences. 
instead of (1.2), where $U$ is a uniformly monotone operator or dual mapping of $X$. The regularized equation (1.3) is difficultly solved by iteration methods, because iterative parameters must satisfy very complex condition (see [3]). To overcome this difficulty [4] we showed another approach of regularization for (1.1). That is the use of linear and strongly monotone operator $B$ instead of $U$ in (1.3), i.e. the use of equation

$$
A(x)+\alpha B x=f_{\delta}
$$

with $\bar{D}(B)=X, S_{0} \subset D(B), S_{0}$ denotes the set of solutions of (1.1), $B$ is linear and

$$
|x|^{2}:=\langle B x, x\rangle \geq m_{B}\|x\|^{2}, x \in D(B), m_{B}>0 \text {. }
$$

Without loss of generality, assume that $m_{B}=1$.

In [4], we presend an iteration method for solving (1.4) with variable parameters of iteration. In this note, we consider another one for (1.4) and the stopage rule for it, when $\delta \rightarrow 0$ and $\alpha$ is shosen such that $\delta / \alpha \rightarrow 0$. Our methods is a generation of [5] and [6] in Hilbert spaces for a Banach spaces.

Below the symbols $\rightarrow$ and $\rightarrow$ denote the strong and the weak convergence for any sequence, respectively.

\section{MAIN RESULTS}

As in [5], we require an addtional condition on $A$ :

For each $N>0 \exists C_{B}(N)>0$ such that

$$
\begin{gathered}
\langle A(x)-A(y), w\rangle \leq C_{B}(N)|x-y||w|, x, y \in D(B) \\
|x|,|y| \leq N, \forall w \in X
\end{gathered}
$$

Note that this condition was considered in [5] under $D(B)=X=H$, is a Hilbert space, and $B$ is bounded.

For finding $x_{\alpha}^{\delta}$, the regularized solution of (1.4), we study the iteration method

$$
x^{n+1}=x^{n}-\rho B^{-1}\left(A_{\alpha}\left(x^{n}\right)-f_{\delta}\right), A_{\alpha}=A+\alpha B,
$$

$\alpha>0$ and $f_{\delta}$ are fixed $\left(x^{0} \in D(B)\right)$.

Theorem 2.1. Let $\langle B x, y\rangle=\langle x, B y\rangle, x, y \in D(B)$ and $0<\rho<\frac{2 \alpha}{\left(\alpha+C_{B}\left(N_{0}\right)\right)^{2}}$, $N_{0}:\left\|x_{\alpha}^{\delta}\right\| \leq N_{0} / 2$. Then

$$
x^{n} \rightarrow x_{\alpha}^{\delta}, \text { as } n \rightarrow+\infty \text {. }
$$


Proof. From (2.2) it implies that

$$
\begin{aligned}
\lambda_{n+1}^{2}: & =\left\langle B\left(x^{n+1}-x_{\alpha}^{\delta}\right), x^{n+1}-x_{\alpha}^{\delta}\right\rangle \\
& =\left\langle B\left(x^{n+1}-x^{n}\right), x^{n+1}-x^{n}\right\rangle+\lambda_{n}^{2}+2\left\langle B\left(x^{n+1}-x^{n}\right), x^{n}-x_{\alpha}^{\delta}\right\rangle \\
& =\lambda_{n}^{2}-2 \rho\left\langle A_{\alpha}\left(x^{n}\right)-A_{\alpha}\left(x_{\alpha}^{\delta}\right), x^{n}-x_{\alpha}^{\delta}\right\rangle \\
& +\rho^{2}\left\langle A_{\alpha}\left(x^{n}\right)-A_{\alpha}\left(x_{\alpha}^{\delta}\right), B^{-1}\left(A_{\alpha}\left(x^{n}\right)-A_{\alpha}\left(x_{\alpha}^{\delta}\right)\right)\right\rangle .
\end{aligned}
$$

Since

$$
\begin{aligned}
& \left\langle A_{\alpha}\left(x^{n}\right)-A_{\alpha}\left(x_{\alpha}^{\delta}\right), x^{n}-x_{\alpha}^{\delta}\right\rangle \geq \alpha\left\langle B\left(x^{n}-x_{\alpha}^{\delta}\right), x^{n}-x_{\alpha}^{\delta}\right\rangle=\alpha \lambda_{n}^{2}, \\
& \left\langle A_{\alpha}\left(x^{n}\right)-A_{\alpha}\left(x_{\alpha}^{\delta}\right), B^{-1}\left(A_{\alpha}\left(x^{n}\right)-A_{\alpha}\left(x_{\alpha}^{\delta}\right)\right)\right\rangle= \\
& =\left\langle A_{\alpha}\left(x^{n}\right)-A\left(x_{\alpha}^{\delta}\right)+\alpha B\left(x^{n}-x_{\alpha}^{\delta}\right), B^{-1}\left(A\left(x^{n}\right)-A\left(x_{\alpha}^{\delta}\right)\right)+\alpha\left(x^{n}-x_{\alpha}^{\delta}\right)\right\rangle= \\
& =\alpha^{2} \lambda_{n}^{2}+2 \alpha\left\langle A\left(x^{n}\right)-A\left(x_{\alpha}^{\delta}\right), x^{n}-x_{\alpha}^{\delta}\right\rangle \\
& +\left\langle A\left(x^{n}\right)-A\left(x_{\alpha}^{\delta}\right), B^{-1}\left(A\left(x^{n}\right)-A\left(x_{\alpha}^{\delta}\right)\right)\right\rangle,
\end{aligned}
$$

we have

$$
\begin{aligned}
& \lambda_{n+1}^{2} \leq \lambda_{n}^{2}-2 \rho \alpha \lambda_{n}^{2}+\rho^{2} \times \\
& {\left[\alpha^{2} \lambda_{n}^{2}+2 \alpha\left\langle A\left(x^{n}\right)-A\left(x_{\alpha}^{\delta}\right), x^{n}-x_{\alpha}^{\delta}\right\rangle+\left\langle A\left(x^{n}\right)-A\left(x_{\alpha}^{\delta}\right), B^{-1}\left(A\left(x^{n}\right)-A\left(x_{\alpha}^{\delta}\right)\right)\right\rangle\right] .}
\end{aligned}
$$

Let $N_{0}$ be a number so that $\left|x_{\alpha}^{\delta}\right| \leq N_{0} / 2$ and $C_{0}=C_{B}\left(N_{0}\right)$. We suppose that the recurrent hypothese $\left|x^{n}-x_{\alpha}^{\delta}\right| \leq N_{0} / 2$, then $\left|x^{n}\right| \leq N_{0}$ and

$$
\left\langle A\left(x^{n}\right)-A\left(x_{\alpha}^{\delta}\right), B^{-1}\left(A\left(x^{n}\right)-A\left(x_{\alpha}^{\delta}\right)\right)\right\rangle \leq C_{0}^{2} \lambda_{n}^{2} .
$$

We have

$$
\begin{aligned}
\lambda_{n+1}^{2} & \leq \lambda_{n}^{2}-2 \rho \alpha \lambda_{n}^{2}+\rho^{2}\left[\alpha^{2} \lambda_{n}^{2}+2 C_{0} \alpha \lambda_{n}^{2}+C_{0} \lambda_{n}^{2}\right] \\
& =\left[1-2 \rho \alpha+\rho^{2}\left(\alpha^{2}+2 \alpha C_{0}+C_{0}^{2}\right)\right] \lambda_{n}^{2} .
\end{aligned}
$$

If we choose $0<\rho<2 \alpha /\left(\alpha+C_{0}\right)^{2}$, then $\theta_{S}=1-2 \rho \alpha+\rho^{2}\left(\alpha+C_{0}\right)^{2}<1$. Therefore, the hypothese is verifies. Consequently, $\lambda_{n} \rightarrow 0$. Hence, the convergence of $x^{n}$ to $x_{\alpha}^{\delta}$ follows from (1.5) and the definition of $\lambda_{n}$.

Remark. In much cases, we can choose the unbounded operator $B$ such that $\|x\|,\|y\| \leq$ $N \rightarrow|x|,|y| \leq N$ in condotion (2.1). In fact, for example $B x(t)=-d^{2} x(t) / d t^{2}+c_{0} x(t)$, $c_{0}>0$, where $D(B)$ is the closure in the norm $W_{q}^{2}, 1<q<2$ of all functions from $C^{2}[0,1]$ satisfying the condition $u(0)=u(1)=0$. Then $B^{-1} v(t)=\int_{0}^{1} g(t, s) v(s) d s$ with

$$
g(t, s)= \begin{cases}u_{1}(t) u_{2}(s), & t \leq s \\ u_{2}(t) u_{1}(s), & t \geq s\end{cases}
$$


where $u_{1}, u_{2}$ are the nontrivial solutions of $B u=0$ such that $u(0)=u(1)=0$. The derivatives are understood in generalized. Then, $|x|^{2}=\langle B x, x\rangle=\int_{0}^{1} c_{0} x(t)^{2} d t=c_{0}\|x\|_{L_{2}[0,1]} \geq$ $c_{0} \tilde{c}_{p}\|x\|_{L_{p}[0,1], 2<p<+\infty}$ with some $\tilde{c}_{p}>0$, since in these cases $L_{p}[0,1]$ is conctinuously embeded in $L_{2}[0,1]$.

For each $\delta>0$, the value $\alpha=\bar{\alpha}=\bar{\alpha}(\delta)$ is chosen that $\delta / \bar{\alpha}(\delta) \rightarrow 0$, then $x_{\bar{\alpha}(\delta)}^{\delta} \rightarrow x_{0}$, the solution of (1.1), as $\delta \rightarrow 0$. In order to approximate the solution $x_{\bar{\alpha}(\delta)}^{\delta}$ of (1.3) with $\alpha=\bar{\alpha}$, we can use the iterative process (2.2). It is important to indicate how many iterations (depending on $\delta$ ) are performed. Choices of $n=n(\delta)$ are also called "stopping rules" in the literature.

We have the result

Theorem 2.2. If the first integer $n=n(\delta)$ satisfying the condition $\left|x^{n+1}-x^{n}\right| \leq$ $a \delta$, where $a>0$, then $x^{n(\delta)} \rightarrow x_{0}$, as $\delta \rightarrow 0$.

Proof. Indeed,

$$
\begin{aligned}
& \left|x^{n+1}-x^{n}\right|=\left\langle B\left(x^{n+1}-x^{n}\right), x^{n+1}-x^{n}\right\rangle \\
& =\rho^{2}\left\langle A_{\bar{\alpha}}\left(x^{n}\right)-f_{\delta}, B^{-1}\left(A_{\bar{\delta}}\left(x^{n}\right)-f_{\delta}\right)\right\rangle \\
& =\rho^{2}\left\langle A_{\bar{\alpha}}\left(x^{n}\right)-f_{0}+f_{0}-f_{\delta}, B^{-1}\left(A_{\bar{\delta}}\left(x^{n}\right)-f_{0}+f_{0}-f_{\delta}\right)\right\rangle \\
& \geq \rho^{2}\left\{\delta^{2}-2 \delta \rho\left|x^{n+1}-x^{n}\right|+\left\langle A_{\bar{\alpha}}\left(x^{n}\right)-A_{\bar{\alpha}}\left(x_{\bar{\alpha}}\right), B^{-1}\left(A_{\bar{\alpha}}\left(x^{n}\right)-A_{\bar{\alpha}}\left(x_{\bar{\alpha}}\right)\right\rangle,\right.\right.
\end{aligned}
$$

where $x_{\bar{\alpha}}: A\left(x_{\bar{\alpha}}\right)+\bar{\alpha} B x_{\bar{\alpha}}=f_{0}$.

Since

$$
\begin{aligned}
& \left\langle A_{\bar{\alpha}}\left(x^{n}\right)-A_{\bar{\alpha}}\left(x_{\bar{\alpha}}\right), B^{-1}\left(A_{\bar{\alpha}}\left(x^{n}\right)-A_{\bar{\alpha}}\left(x_{\bar{\alpha}}\right)\right\rangle \bar{\alpha}\left|x^{n}-x_{\bar{\alpha}}\right|\right. \\
& +\bar{\alpha}\left\langle A\left(x^{n}\right)-A\left(x_{\bar{\alpha}}\right), x^{n}-x_{\bar{\alpha}}\right\rangle \geq \bar{\alpha}^{2}\left|x^{n}-x_{\bar{\alpha}}\right|
\end{aligned}
$$

we have

$$
\left|x^{n+1}-x^{n}\right|^{2} \geq \bar{\alpha}^{2} \rho^{2}\left|x^{n}-x_{\bar{\alpha}}\right|^{2}-2 \rho \delta\left|x^{n+1}-x^{n}\right|-\rho^{2} \delta^{2} .
$$

Therefore,

$$
\left|x^{n}-x_{\bar{\alpha}}\right| \leq \frac{2 \delta}{\bar{\alpha}}
$$

This means that $x^{n} \rightarrow x_{0}$, as $\delta \rightarrow 0$.

\section{REFERENCES}

1. J. I. Alber, On solution of nonlinear problem involving monotone operators in Banach spases, Sibirskii Math. J., 16 (1975) 3-11 (Russian).

2. J. I. Alber and I.P. Riazanseva, On the solution of the nonliear equations with monotone and discontinuous operators, URSS Differential Equations, 15 (2) (1979) 331-342 (Russian). 
3. J. I. Alber and A.I. Notik, The geometrical characteristics of the Banach spaces and the method od approximation for the nonlinear problems, URSS Dokl. Acad. Nauk, 276 (5) (1984) 1033-1037 (Russian).

4. Nguyen Buong, Linear and strongly monotone operator in regularization for ill-posed problems, Proceedings of the NCST of Vietnam, 7 (1995), 9-18.

5. M. Sibony, Analyse numerique III, Iteration et approximations, Hermann, ed. des Sciences et des Artts, 476 p, 1988.

6. I. P. Ryazatseva, On the stopage rule in solution of nonlinear ill-posed problems, AIT, 10 (1985) 27-30.

Institute of Information Technology

Nghia Do, Tu Liem, Hanoi.

Received: August 14, 1997 